Conclusion Although there has been substantial focus up to now on the role that increased mortality amongst the oldest groups has played in the recent life expectancy trends, more attention needs to be paid to the role that the slowdown in improvement for circulatory causes across a much wider age group has played, as well as the importance of rising drugrelated deaths in younger adults. Future research should seek to explain the changes in mortality trends for all age groups and causes of death and policymakers should act to protect the social security system and public services that are likely to be part of the causes of the recent trends.

\section{P38 RISK AND PROTECTIVE FACTORS FOR PSYCHOTIC EXPERIENCES IN ADOLESCENCE: A POPULATION-BASED STUDY}

${ }^{1} \mathrm{E}$ McMahon*, ${ }^{1,2} \mathrm{P}$ Corcoran, ${ }^{3} \mathrm{H}$ Keeley, ${ }^{4} \mathrm{M}$ Clarke, ${ }^{5} \mathrm{H}$ Coughlan, ${ }^{6} \mathrm{D}$ Wasserman, ${ }^{7,8} \mathrm{C}$ Hoven, ${ }^{6} \mathrm{~V}$ Carli, ${ }^{9,10} \mathrm{M}$ Sarchiapone, ${ }^{5} \mathrm{M}$ Cannon. ${ }^{1}$ National Suicide Research Foundation, University College Cork, Cork, Ireland; ${ }^{2}$ School of Public Health, University College Cork, Cork, Ireland; ${ }^{3}$ Child and Adolescent Mental Health Services, Health Service Executive, Cork, Ireland; ${ }^{4}$ Depts of Psychology and Psychiatry, Royal College of Surgeons in Ireland, Dublin, UK; ${ }^{5}$ Dept of Psychiatry, Royal College of Surgeons in Ireland, Dublin, UK; ${ }^{6}$ National Centre for Suicide Research and Prevention of Mental III-Health (N, Karolinska Insitute, Stockholm, Sweden; ${ }^{7}$ Child and Adolescent Psychiatry, Columbia University, New York, USA; ${ }^{8}$ Child and Adolescent Psychiatry, New York State Psychiatric Institute, New York, USA; ${ }^{9}$ Dept of Medicine and Health Science, University of Molise, Campobasso, Italy; ${ }^{10}$ National Institute for Health, Migration and Poverty, Rome, Italy

\subsection{6/jech-2019-SSMabstracts. 189}

Background Psychotic experiences (PEs) are reported by a significant minority of adolescents and are associated with the development of schizophrenia and other psychiatric disorders in adulthood. Few modifiable protective factors have been identified to date. The aims of this study were to examine associations between a range of psychopathological, adverse life event, coping and social support factors and PEs in a general population sample of Irish adolescents.

Methods Cross-sectional data were drawn from the Irish centre of the Saving and Empowering Young Lives in Europe (SEYLE) study (German Clinical Trials Registry, DRKS00000214). Students were administered a classroombased self-report questionnaire and 973 adolescents, of whom 522 (53.6\%) were boys, participated. Psychotic experiences were assessed using the 7-item Adolescent Psychotic Symptom Screener.

Results Of the total sample, $81(8.7 \%)$ of the sample were found to be at risk of PEs. A wide range of factors from adverse life event, lifestyle and mental health domains had crude associations with PEs, while parental support was associated with lower prevalence of PEs. In multivariate analysis, independent associations were found between PEs and the number of adverse life events experienced (OR: 1.61; CI: $1.29-2.02 ; \mathrm{p}<0.0005)$ as well as maladaptive/pathological internet use (OR: 2.60; CI: 1.15-5.89; $\mathrm{p}=0.02$ ). Positive parental support was associated with reduced risk of PEs after adjustment for established risk factors (OR: 0.40; CI: $0.18-$ $0.90 ; p=0.03)$ and so offers a potential protective role.

Conclusion These findings can inform the development of optimal interventions for adolescents at risk of psychopathology and their families.

\section{P39 MEAT, FRUIT AND VEGETABLE CONSUMPTION IN SUB-SAHARAN AFRICA: A SYSTEMATIC REVIEW AND META-REGRESSION}

${ }^{1}$ DO Mensah*, ${ }^{1} 0$ Oyebode, ${ }^{1}$ RA Nunes, ${ }^{2}$ R Lillywhyte. ${ }^{1}$ Health Sciences Division, Warwick Medical School, University of Warwick, Coventry, UK; ${ }^{2}$ School of Life Sciences, University of Warwick, Coventry, UK

\subsection{6/jech-2019-SSMabstracts. 190}

Background The dietary choices we make affect our personal health and have consequences for the environment, both of which have serious implications for the 2030 Sustainable Development Agenda. There is a strong consensus that cutting on meat and dairy products in favour of fruit and vegetables and other plant-based diets would offer dual health and environmental benefits. In global reviews, the literature on meat, fruit, and vegetable consumption in sub-Saharan Africa (SSA) is limited. It is therefore essential to quantify meat, fruit, and vegetable consumption in sub-Saharan African populations.

Methods We systematically searched six databases to identify studies reporting meat, fruit and/or vegetable consumption in sub-Saharan African populations. Using STATA SE version 15, random effects meta-regression analyses were used to test the effect of year of data collection and method of data collection on population meat, fruit, and vegetable consumption. We also tested any association between age, sex, urban/rural residence or a country's economic development, and population intake of meat, fruits and/or vegetables.

Results Richer SSA countries were likely to consume more meat $(B=36.76, p=0.04)$ and vegetables $(B=43.49, p=0.00)$ than poorer countries. Vegetable intake has increased dramatically over the last three decades from $\approx 10 \mathrm{~g}$ to $\approx 110 \mathrm{~g}$ $(\beta=4.43, p=0.00)$. Vegetable $(\beta=-25.48, p=0.00)$ consumption was higher in rural than urban residents. Although the trend of meat consumption has gone up $(\approx 25 \mathrm{~g}$ to $\approx 75 \mathrm{~g})$, the trend is non-significant $(\beta=0.63$, N.S.). Daily average per capita meat consumption was however above recommended $70 \mathrm{~g}$, while fruit and vegetable consumption remain below WHO's recommendation, though consumption of both fruit and vegetable has increased over the last three decades. No clear differences in consumption were noticed between sexes.

Conclusion While dietary changes in SSA may offer the large absolute benefits, consideration of the magnitude of dietary change, particularly increasing meat consumption, will need to occur to ensure policy and interventions support the reduction of under-nutrition and micronutrient deficiencies without worsening NCD prevalence and environmental impacts.

\section{P40 PREDICTING TYPE 2 DIABETES DEVELOPMENT AMONG PATIENTS IN GENERAL PRACTICE - A PROSPECTIVE ANALYSIS COMPARING METABOLIC SYNDROME DEFINITIONS AND COMPONENTS}

SR Millar*, CM Phillips, JM Harrington, IJ Perry. School of Public Health, University College Cork, Cork, Ireland

\subsection{6/jech-2019-SSMabstracts.191}

Background A definition of metabolic syndrome (MetS) has been recommended as a tool to help identify individuals at risk of developing type 2 diabetes. However, an agreed 
protocol for defining MetS does not exist and some studies have shown MetS definitions to be inferior at predicting diabetes compared to a single measurement of fasting glucose. In this study we examined the ability of five proposed MetS definitions to discriminate incident cases in order to determine whether MetS more accurately predicts type 2 diabetes.

Methods This was a prospective study involving a random sample of 1,754 men and women aged 46-73 years. Receiver operating characteristic curve and net reclassification improvement (NRI) analyses were used to evaluate the ability of MetS definitions and components to accurately classify high-risk subjects.

Results A model including proposed MetS components displayed a significantly $(\mathrm{P}=0.02)$ higher area under the curve (AUC) to discriminate diabetes (AUC $=0.90$, 95\% CI: $0.87-$ 0.93 ) compared to fasting glucose alone $(\mathrm{AUC}=0.88,95 \% \mathrm{CI}$ : 0.83-0.92). Models using the European Group for the Study of Insulin Resistance MetS criterion, and which included glucose as a mandatory component, demonstrated significant overall NRI when compared to recommended and optimal fasting glucose cut-offs. A final model had a sensitivity of 0.91 and a specificity of 0.73 .

Conclusion In this population there is evidence that a combination of MetS components may help predict diabetes beyond that which is measured by glucose alone. Proposed MetS definitions should include fasting glucose as a mandatory component.

\section{\begin{tabular}{l|l} 
P41 DEVELOPING A HEALTHY LIFESTYLE INDEX FOR \\
\hline
\end{tabular} ASTHMA AND ALLERGY PREVENTION IN CHILDHOOD}

${ }^{1} \mathrm{E}$ Morales*, ${ }^{2} \mathrm{D}$ Strachan, ${ }^{3} \mathrm{I}$ Asher, ${ }^{3} \mathrm{P}$ Ellwood, ${ }^{4} \mathrm{~N}$ Pearce, ${ }^{1} \mathrm{~L}$ Garcia-Marcos. ${ }^{1} / \mathrm{MIB}$ Arrixaca Biomedical Research Institute of Murcia, Murcia, Spain; ${ }^{2}$ Population Health Research Institute, St George's, University of London, London, UK; ${ }^{3}$ Department of Paediatrics: Child and Youth Health, University of Auckland, Auckland, New Zealand; ${ }^{4}$ Department of Medical Statistics, Faculty of Epidemiology and Population Health, London School of Hygiene and Tropical Medicine, London, UK

\subsection{6/jech-2019-SSMabstracts. 192}

Background Although asthma is currently the most common chronic disease in childhood worldwide, it is not the key focus of prevention strategies. Diverse modifiable parental and child lifestyle factors has been individually associated with the risk of asthma and allergy symptoms in childhood; however, the quantification of their joint effect is lacking. A healthy lifestyle index (HLI) was developed to examine the combined effect of modifiable lifestyle factors on asthma, rhinoconjunctivitis, and eczema using data from the multicentre International Study of Asthma and Allergies in Childhood (ISAAC) Phase Three. We used this multidimensional lifestyle approach to estimate the impact of potential prevention strategies (mainly recommended for non-respiratory diseases) upon the population burden of asthma, rhinitis, and eczema in childhood.

Methods Information on symptoms of asthma, rhinoconjunctivitis, eczema and several lifestyle factors was obtained from children aged 6-7 years through written questionnaires. The HLI combined five lifestyle factors: no parental smoking, child's adherence to Mediterranean diet, child's healthy body mass index, high physical activity and non-sedentary behaviour. The HLI was modelled as a continuous and categorical variable with four categories ( 0 or 1 factor, 2 factors, 3 factors, and 4 or 5 factors), using 0 or 1 factor as the reference category. The association between the HLI and risk of asthma, rhinoconjunctivitis, and eczema was evaluated using multilevel mixed-effects logistic regression models. To estimate the proportion of cases in the entire study population that could be prevented had all children been following four or all five healthy lifestyle factors (i.e. lowest-risk exposure group), we derived centre-specific population-attributable risk fractions.

Results Data of 70795 children from 37 centres in 19 countries were analysed. Each additional healthy lifestyle factor was associated with a reduced risk of current wheeze $(\mathrm{OR}=0.87$, 95\% CI 0.84-0.89), asthma ever $(\mathrm{OR}=0.89$, 95\% CI 0.87-0.92), current symptoms of rhinoconjunctivitis $(\mathrm{OR}=0.95,95 \%$ CI 0.92-0.97), and current symptoms of eczema $(\mathrm{OR}=0.92,95 \%$ CI $0.92-0.98)$. Theoretically, if associations were causal, a combination of four or five healthy lifestyle factors would result into a reduction up to $16 \%$ of asthma cases (ranging from 2.7 to $26.3 \%$ according to region of the world).

Conclusion These findings should be interpreted with caution given the limitations to infer causality from cross-sectional observational data. Efficacy of interventions to improve multiple modifiable lifestyle factors to reduce the burden asthma and allergy in childhood should be assessed.

\section{P42 RECRUITMENT STRATEGIES AND LESSONS FROM THE WE CAN QUIT2 TRIAL - A SMOKING CESSATION COMMUNITY-BASED CLUSTER RANDOMISED CONTROLLED TRIAL FOR WOMEN LIVING IN DISADVANTAGED AREAS OF IRELAND}

${ }^{1} \mathrm{E}$ Burke*, ${ }^{1} \mathrm{~N}$ O'Connell, ${ }^{1} \mathrm{C}$ Darker, ${ }^{2} \mathrm{~J}$ Vance, ${ }^{3} \mathrm{~N}$ Dougall, ${ }^{4} \mathrm{~L}$ Bauld, ${ }^{1} \mathrm{C}$ Hayes. ${ }^{1}$ Public Health and Primary Care, Trinity College Dublin, Dublin, Ireland; ${ }^{2}$ Health Promotion, Irish Cancer Society, Dublin, Ireland; ${ }^{3}$ School of Health and Social Care, Edinburgh Napier University, Edinburgh, UK; ${ }^{4}$ The Usher Institute, University of Edinburgh, Edinburgh, UK

\subsection{6/jech-2019-SSMabstracts. 193}

Background Recruitment is a challenge in community-based randomised controlled trials (RCTs). Evidence on recruitment efforts are not routinely available although they help predict rates and manage risk. This study aims to describe recruitment strategies used in the We Can Quit2 (WCQ2) study, and successes and barriers.

Methods WQC2 trial is a pilot, pragmatic, parallel-group cluster RCT delivered to women living in disadvantaged areas in Ireland. It tests the WCQ2 programme, a smoking-cessation behavioural support intervention delivered through 12-weekly group sessions, with free Nicotine Replacement Therapy, against a form of usual care.

Four Local Area Advisory Groups (LAGs) were established which consist of local area stakeholders, for example the Irish Cancer Society, the HSE, community organisations, and community workers. LAGs identified four matched pairs of districts within their region (eight clusters) from which eligible women could be recruited. LAGs and the study researcher recruited participants. The recruitment target was 24-25 women in each cluster (97 per arm; 194 in total). Consent was obtained prior to cluster randomisation.

Results Participants were recruited through a variety of methods including leafleting, posters in local shops and community services, information stands at local public events and in facilities like creches, and through traditional and social media, e.g. local radio and targeted Facebook advertisements. The trial was promoted at community employment schemes, and parenting groups. 